\title{
An Edge-endpoint-based Configurable Hardware Architecture for VLSI Layout Design Rule Checking
}

\author{
ZHEN LUO $^{\mathrm{a}, *}$, MARGARET MARTONOSI ${ }^{\mathrm{a}}$ and PRANAV ASHAR \\ ${ }^{a}$ Department of Electrical Engineering, Princeton University, Princeton, NJ 08544; ${ }^{\mathrm{b}} \mathrm{NEC} C C R L$, Princeton, NJ 08540
}

(Received 1 February. 1999; In final form 1 October 1999)

\begin{abstract}
Previous efforts to build hardware accelerators for VLSI layout Design Rule Checking (DRC) were hobbled by the fact that it is often impractical to build a different rulechecking ASIC each time design rules or fabrication processes change. In this paper, we propose a configurable hardware approach to DRC. It can garner impressive speedups over software approaches, while retaining the flexibility needed to change the rule checker as rules or processes change.

Our work proposes an edge-endpoints-based method for performing Manhattan geometry checking and a general scalable architecture for DRC. We then demonstrate our approach by applying this architecture to a set of design rules for MOSIS SCN4N_SUB process. We have implemented several design rule checks within a single Xilinx XC4013 FPGA and demonstrated overall speedups in excess of 25X over software methods. We have used a Compaq Pamette board to do the hardware prototyping and have achieved a clock rate of $33 \mathrm{MHz}$.
\end{abstract}

Keywords: Design Rule Checking, scanline, configurable hardware, FPGA, Pamette

\section{INTRODUCTION}

Over the past several decades, integrated circuit die sizes have increased dramatically and simultaneously the smallest possible features on these dies have become much smaller as well. As a result, Design Rule Checking (DRC), which checks a VLSI layout's features for compliance with width and spacing rules, has become more and more time-consuming and compute-intensive.
In the past, several approaches for hardware DRC accelerators have been proposed $[1,3,5]$. The main difficulty with prior, custom-hardware proposals has been their inflexibility. Fabrication processes evolve over time, with new layers or width/ spacing rules being introduced. As such, design rules checkers implemented in hardware must be re-designed and rebuilt to address each set of changes. An ASIC-based DRC for a particular set of design rules would be used too few times before

\footnotetext{
*Corresponding author.
} 
new rules and therefore a new ASIC are needed. Past hardware DRC approaches avoided this redesign cycle by accelerating only the basic primitives of DRC, not the specific rule checks. The drawback to such approaches is that they accelerate only a portion of the DRC and do not adequately address the true compute bottlenecks.

Our proposal notes that while design rules do change over time and vary between fabrication lines, their fundamental form remains similar. Thus, our goal is to design a general-purpose skeleton for DRC that applies to nearly all fabrication design rules, and then also to tailor the rule-checking hardware for a particular fabrication process. Tailoring the rule-checks to an individual process allows for much better speedups and takes advantage of the inherent flexibility of configurable hardware. At the same time, the reconfigurations are likely to be infrequent enough (every $6-12$ months) that the FPGA reconfiguration times have essentially no impact on performance.

This paper presents our configurable hardware accelerator for design rule checking. In order to take the best advantage of the special characteristics of FPGAs, we have also developed a new DRC methodology that we present here. This edgeendpoint-based approach reduces the storage and sorting requirements for processing the layout files compared to prior work. This makes the approach particularly amenable to FPGAs and also reduces the cost considerably. Our evaluations of the DRC architecture indicate that it offers speedups of $25 \mathrm{X}$ or more over software approaches.

The remainder of the paper is structured as follows. Section 2 gives background on design rule checking and surveys prior work on its hardware acceleration. Section 3 then gives an overview of our edge-endpoints-based approach, while Section 4 focuses in more detail on the resulting hardware DRC architecture. In Section 5, we offer a case study on how to apply our architecture to a specific fabrication technology example: the MOSIS SCN4M_SUBM process. Based on this example, Section 6 presents synthesis results, performance evaluation and I/O requirement analysis. In Section 7, we present the hardware prototyping with a Compaq Pamette FPGA board. Finally, Section 8 discusses the future work, and Section 9 presents our conclusions.

\section{DESIGN RULE CHECKING: BACKGROUND AND RELATED WORK}

Design Rule Checking takes as input a low-level description of the mask layers and features required for a particular VLSI design. Such data is produced by the CAD tool with which the layout was created. The goal of DRC is to identify places in the VLSI design in which design rules, such as the spacing between two features or the width of a wire, have been violated. Specifying design rules in terms of a parameterized width factor, typically referred to as lambda, sometimes allows the same design rules to be used as the feature size of the process changes. In spite of this, design rules change frequently and many fabrication processes, particularly in the submicron domain, will have subtly different design rules. Section 5 discusses the MOSIS SCMOS design rules on which our current implementation is based.

There are two major types of design rule checking methods. Bitmap methods were widely used in early approaches [2-4]. The layout is rasterized into a grid of square cells, with each mask layer represented by a separate bit in each cell. Bit maps are attractive because of the simplicity of some operations such as Boolean operations (AND of two masks, for example) and space/width checking. Many bit map approaches are based on Baker's algorithm [2]. This algorithm uses a $3 \times 3$ window to do the checking when minimum width is 2 and a $4 \times 4$ window to do the checking when minimum width is 3 . There are, however, disadvantages to bitmap approaches. The first one is that the bitmap approach requires processing a large amount of data; this requires large amounts of memory bandwidth and high parallelism in 
order to produce results with acceptable performance. In [3], special hardware "expands" the layer data on-the-fly into bitmaps to shrink intermediate storage requirements; nonetheless, the total amount of data being processed remains the same. The second disadvantage is that in a design system where the grid spacing (i.e., the minimum feature spacing) is much smaller than the minimum feature size, we need a much larger window size to check for width or spacing errors. If this is the case, significant time will be spent comparing error templates with windows [4].

Edge-based approaches, on the other hand, use edges to represent regions in each mask layer. This helps to reduce the amount of data needed in general and is less dependent on the mask resolution. Kane and Sahni [5] proposed a one-dimensional systolic architecture for design rule checking for Manhattan structures, with only horizontal and vertical features. The edge files are divided into horizontal edges and vertical edges and each set is processed independently. Horizontal edges are checked for vertical width errors and horizontal spacing errors; vertical edges are checked for horizontal width errors and vertical spacing errors. The main disadvantage of this approach is that it requires non-deterministic amounts of hardware.

Most of the edge-based approaches instead use variations on scanline algorithms $[1,6]$. The mask layer data are transformed into an edge file that contains all non-vertical edges in the mask. Each edge is described by a pair of points $\left(X_{\min }, Y_{\min }\right)$ and $\left(X_{\max }, Y_{\max }\right)$ along with an Orientation field indicating if the edge borders the region from above or below. Additional layer information is needed when multiple layers are handled together. The edges are sorted in a canonical order, by nondecreasing order of slope within $y_{\min }$ and $x_{\min }$, before processing. A vertical scanline sweeps horizontally across the whole mask and only stops at the $X$-coordinates of all the edge endpoints. Edges that intersect the current scanline are then processed. This approach requires less hardware than previously discussed approaches.

\section{AN EDGE-ENDPOINT-BASED DRC APPROACH}

The method we propose is focused on DRC for Manhattan structures. DRC with non-Manhattan structures increases complexity considerably. Since wires can intersect at arbitrary angles, it requires multiplications and divisions (generally implemented in floating point) to calculate intersecting points. This reduces its applicability to configurable hardware. For this reason, we focus here on accelerating DRC for Manhattan structures; in designs containing non-Manhattan regions, we will separately run software DRC on these regions, while using configurable hardware for the strictly Manhattan regions.

We use a variant of the scanline algorithm discussed in the previous section, rather than directly implementing the previously-described algorithm, for several reasons described below:

(a) First, edge-based processing leads to a fairly wide datapath. For Manhattan structures, we need at least 3 coordinate fields $\left(X_{\min }, X_{\max }\right.$ and $Y$ ) for each edge: two $X$-coordinates for beginning and ending points and one $Y$-coordinate for the edge. If each coordinate takes 18 bits as we use now, we need at least 54 bits just to store these three coordinates. This imposes constraints on FPGA size and overall system cost and performance.

(b) Second, some design rules require the generation of intermediate (or "working") layers that are Boolean transformations of true layers. When an intermediate layer is generated in an edge-based algorithm, its edge file is not in canonical order, since the edge with the smallest $X$-coordinate of its ending point leaves the pipeline first. Thus an intermediate sort is needed [1]. This adds complexity to the control and data flow in the hardware system and extra hardware cost for temporary storage. Our variant algorithm avoids the need for these costly intermediate sorts. 
(c) Third, we know of no prior work that fully addresses the edge-reconciliation problem [7]. A general method to obtain computational parallelism in DRC is to cut each mask layer into pieces and process each piece individually. This is possible both in bit-map approaches and edge-based algorithms. Spurious width/ spacing errors, however, could be generated by the DRC system because some regions are split into pieces. Sewing regions back together imposes non-trivial performance (especially for edge-based approaches) when the mask is divided into many small pieces.

For the above reasons, we propose a new approach that is amenable to configurable hardware and capable of solving the problems in (b) and (c).

Our approach assumes a "virtual" scanline that passes over the mask layers, checking relevant design rules as it goes. As the scanline passes over the mask, it takes note of features like edges and their endpoints. In order to check vertical spacings or widths, the scanline will be oriented vertically, as shown in Figure 1. In order to check horizontal spacings or widths, the mask is rotated by 90 degrees counterclockwise and the scanline passes over it again. In reality, since we are focused on design rule checking before fabrication, no true scanning occurs, but rather the features of the mask are extracted from the layout file generated by the CAD tool.

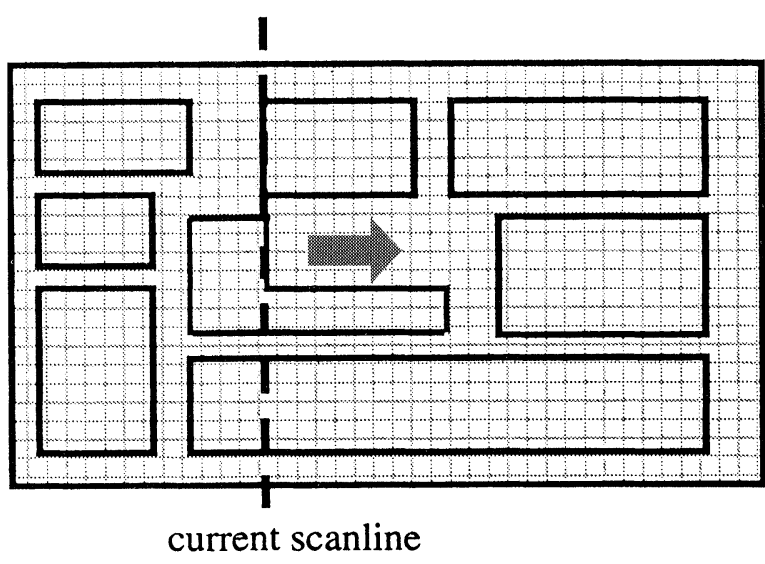

FIGURE 1 Example mask layer with scanline.
Our method uses an edge-endpoint-based representation. That is, we categorize all the edges in a mask layer into horizontal edges and vertical edges and their endpoints are processed separately. By doing this, we can solve the problems mentioned above. From this point on, we will only talk about how to process the endpoints associated with horizontal edges. Endpoints associated with vertical edges will be processed in exactly the same way after the mask is rotated.

\subsection{Representation}

An endpoint is represented in the following format:

\section{\begin{tabular}{|l|c|c|c|c|c|}
\hline Y-coordinate & Layer & O & D & P & X-extension \\
\hline
\end{tabular}}

FIGURE 2 Endpoint format.

The $Y$-coordinate field represents the $Y$-coordinate of the edge endpoint. The Layer field indicates the layer this endpoint belongs to. The $O$ bit indicates the orientation of the edge with which the endpoint is associated. $O=$ ' 0 ' if the edge is a forward edge, i.e., the edge borders the region from above; $O=$ ' 1 ' if the edge is a backward edge, i.e., the edge borders the region from below. The $D$ bit indicates the direction of the current endpoint. $D=$ ' 0 ' if the endpoint is the starting endpoint of the associated edge. $D=$ ' 1 ' if the endpoint is the ending endpoint of the associated edge. The $P$ bit indicates if the endpoint has been processed before. For the endpoints generated from mask layer data, $P={ }^{\prime} 0$ ' and $X$-extension $=0$. We will further discuss the $P$ bit and talk about $X$ extension fields later in this section.

We maintain the endpoints in a canonical order. All the endpoints are sorted in increasing order by $X$-coordinate, $Y$-coordinate, layer and orientation. Two endpoints in the initial edge file "cancel" if they only differ in the $D$ bit. That is, after endpoints are sorted, they are divided into groups with the same $X$-coordinate. Within each group they are sorted in increasing order by their first three fields. Note in the endpoint representation, there 
is no $X$-coordinate information. Instead, a special endpoint of layer 0 is created to carry the $X$-coordinate by its $Y$-coordinate field and inserted before each endpoint group of same $X$-coordinate.

\subsection{Scanline Maintenance}

When the scanline is at a particular $X$-coordinate $X_{c}$, we categorize and sort pending edges as follows:

(1) $X_{\min }=X_{c}$, i.e., the edge starts from this scanline.

(2) $X_{\max }=X_{c}$, i.e., the edge ends at this scanline.

(3) $X_{\min }<X_{c}<X_{\max }$, i.e., the edge intersects this scanline.

Endpoints from the first two categories are called new endpoints with $P=$ ' 0 ' since they have never been processed before. The points of the third category, however, are "inherited" from the previous scanline. Each time a category-1 edge startpoint is processed, it is passed along to the next scanline. This point inherits all the information from the starting endpoint except that now $P=$ ' 1 ' since they have been processed before. We refer to these points as old points. Old points are removed from the scanline when they meet the ending endpoints (category 2 ) of the edge.

\subsection{Scanline Processing}

Endpoints of the current scanline, after being merge-sorted with old points inherited from the previous scanline, stream into the processing unit as shown in Figure 3. Generally speaking, there are several processing steps:

\subsubsection{Selecting True Points}

In the input data from the CAD tool, there can be overlapped and coincident regions in some layers. These regions should be logically ORed to get the final region profile (see Fig. 4). A point, whether it is an old point or a starting/ending endpoint of an edge, is called a true point, following Lauther's

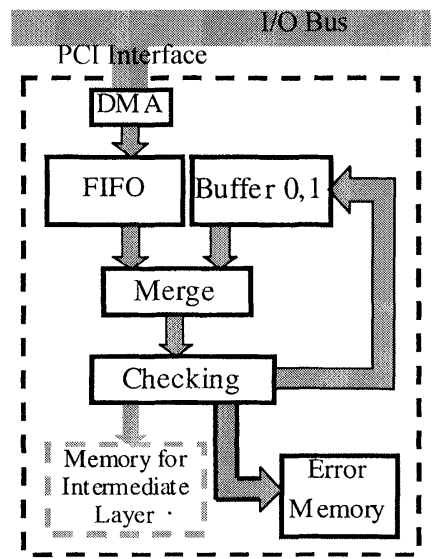

FIGURE 3 Basic processing unit (BPU).

terminology [11], if it is on the boundary of the final region. A true point is called a true endpoint, if it is one of the endpoints of the final region. Thus we need to select out the true points and only consider them in the design rule checking.

To help select the true points, we keep two counters for each layer: $L C$ and $R C$. When a point enters the processing unit, the counters corresponding to its layer are updated in the following way:

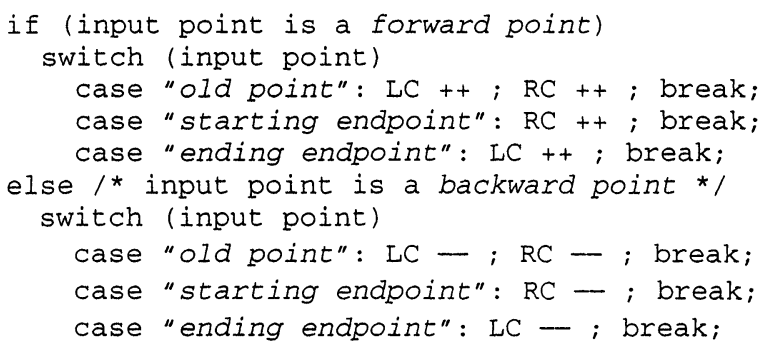

Figure 4 illustrates the counter update process. Point $C$ is a forward old point, thus both $L C$ and $R C$ are incremented. Point $A$ is a backward starting endpoint, thus $R C$ is decremented. Point $B$ is a backward ending endpoint, thus $L C$ is decremented.

$L C$ indicates if the left side just below the current position is inside or outside the final region. If $L C>0$, the left side below the current position is inside the final region; if $L C=0$, the left side below the current position is outside the final 


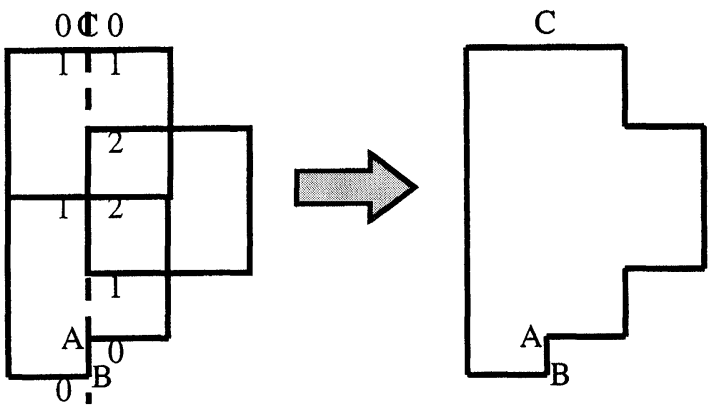

FIGURE 4 Selecting true points.

region. $L C$ should never be less than zero, however, unless there is an error in the endpoint file. $R C$ similarly indicates the situation on the right side of current position. In Figure 4, we can see that both the left side and right side below point $C$ is inside the region, since $L C>0$ and $R C>0$. At point $A$, the left side below it is inside the region and the right side below it is outside the region, since $L C>0$ and $R C=0$. Both the left side and right side of point $C$ is outside the region, as indicated by $L C=0$ and $R C=0$.

Special care has to be taken when dealing with coincident points. In considering two coincident points, the forward point of the two should always be sent to the processing unit; this lets us know that these two regions "overlap". In our approach, this ordering is guaranteed by the canonical order of the endpoints.

Thus a point is a true point if it changes the "inside the region" or "out of the region" status on either or both sides of the scanline. In Figure 4, $A, B$ and $C$ are the true points of the current scanline. A true point is a true endpoint if it changes the "inside the region" or "outside the region" status only on one side of the scanline. In Figure $4, A$ and $B$ are true endpoints and $C$ is not.

\subsubsection{Checking Design Rules}

Most design rule checking problems boil down to different instances of width checking. Space checking of a specific mask layer, for example, is equivalent to width checking on the inverse of such a mask. Other types of checking, such as minimum overlap, can all be transformed to width-checking in a similar way. This does not mean, however, that we have to transform the design to these intermediate forms before checking these rules. Instead, when multiple layers are present, all the rules that are associated with these layers can be checked simultaneously, as we will see in the example given later in this section.

Some design rules in modern fabrication processes, however, are hard or impossible to handle with the above approach. Design rules like checking the area range of a certain layer cannot be converted to width checking by some logic operations. To check these design rules, we need additional hardware or software assistance. Fortunately, the number of these hard-to-handle rules is very small by comparison to the total number of design rules for a specific fabrication process. For example, all the design rules for MOSIS SCMOS_ SUBM fabrication process can be converted to width checking. In our current implementation, we resort to software to check these rare cases of design rules that cannot be handled by width checking while maintaining a relatively low hardware requirement.

Design rule checking is also a local problem in that only "adjacent" regions interact with each other. In a bitmap approach, this locality is utilized by means of pattern matching on a twodimension window. In an edge based approach, this locality is utilized by processing only adjacent true edges along the scanline, so is in our edge endpoint based approach. Here adjacency not only refers to the adjacency within one layer, but among different layers as well. We define the point and edge adjacency as follows:

Definition A true point $A$ from layer $I$ is said to be adjacent to a true point $B$ from layer $J$ ( $I$ and $J$ could be the same) if no other point from layer $I$ or layer $J$ is inside or on the boundary of the rectangle formed by these two points. Edge $l$ and $m$ are said to be adjacent if $\exists$ a true point $A \in l$ and a true point $B \in m, A$ and $B$ are adjacent. 
COROLlary Two adjacent true points along any scanline are adjacent. Thus the two edges that contain these two points are adjacent.

Proof Consider the degenerated rectangle formed by these two points.

Our definition of adjacency allows us to recognize that design rule violations on these points can be recognized by comparing $A$ and $B$ only. Since $C$ is further from $A$ than $B$ is, any design rule it violates with respect to $A$ will also be violated by $B$. (Different adjacencies will be relevant for different layers and design rules.) Therefore, in our implementation, only the latest point of each layer along the scanline is stored in the $Y$ coordinate register of that layer for comparison with current true point.

Once a true point from a layer is determined, the checker circuit for each design rule needs: (1) the $Y$ coordinate differences between this point and the latest points of each layer, and (2) the orientation of this point and the orientations of the latest points of each layer to find possible errors.

Figure 5 shows a block diagram of a DRC system for $N$ and $P$ diffusion layers. Here, there are rules for minimum width of an $\mathrm{N}$-diffusion, minimum width of a $P$-diffusion, minimum spacing between two $N$-diffusions, minimum spacing between two $P$-diffusions and minimum spacing between an $N$-diffusion and a $P$-diffusion. However, not all the rule checkers are activated when a true point from $N$ or $P$ layer is filtered out. For example, when a true point of $N$-diffusion layer is filtered out, the rule checker for minimum width of an $N$-diffusion is activated only if the previous $N$

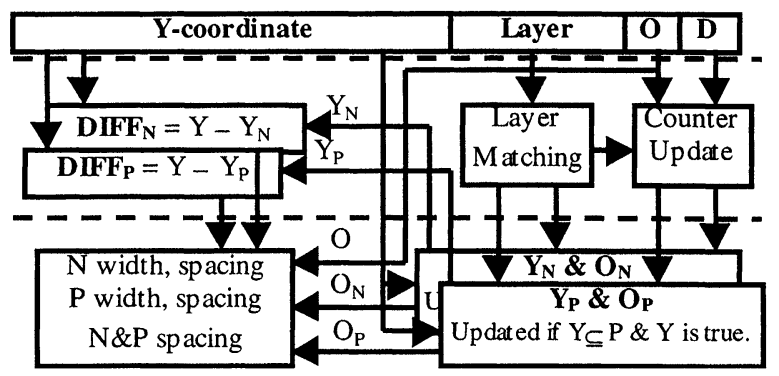

FIGURE 5 Checking unit for $N$ and $P$ layers. point was forward $\left(O_{N}=0\right)$ and the current $N$ point is backward $(O=1)$. The rule checker for minimum spacing of an $N$ diffusion is activated only if the previous $N$ point was backward $\left(O_{N}=1\right)$ and the current $N$ point is forward $\left(O_{N}=0\right)$. The rule checker on spacing between an $N$ diffusion and a $P$ diffusion is activated only if the previous $P$ point was backward $\left(O_{P}=1\right)$ and the current $N$ point is forward $\left(O_{N}=0\right)$. Similar activation criteria apply for all the other design rule checkers.

\subsubsection{Handling Other Cases Using Virtual Edges}

The design rule checking hardware described above is able to find all the design rule errors along a scanline. Thus if all the vertical and horizontal scanlines are processed, we should be able to find all the errors along any vertical or horizontal line. Our design would be complete now except for a special type of error, shown in Figure 6.

In Figure 6, the central box has spacing errors with the surrounding four boxes. That is, if we expand the central box by the minimum space $d$, the expanded region (the dotted box in the center) will intersect all four boxes. (Here we use Mahattan distance instead of Euclidean distance. For design rules that use Euclidean distance, we can treat them in the same way as described below, except we will use a table lookup to get the Euclidean distance

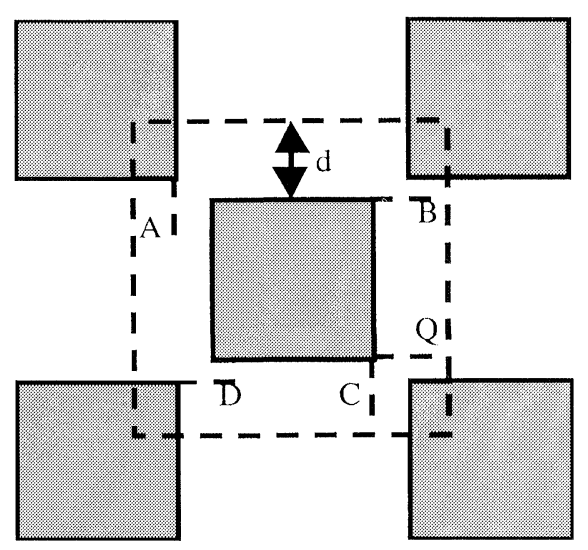

FIGURE 6 Virtual edges. 
when we check for design rule violations.) The case in Figure 6 escapes our DRC hardware thus far since no scanline will cross both the edges from the central box and the four boxes around it. Thus a natural way to solve this problem in our existing hardware framework is to extend some edges of the central box or the boxes around it by $d$, so that scanline processing will be able to find the error. We call these extended edges virtual edges. Each point on a virtual edge is a virtual point.

A virtual point inherits the $Y$-coordinate, Layer and Orientation information from the edge that generates it. Virtual points are old points, so $P=$ ' 1 '. We use the $D$ bit to differentiate virtual points and normal old points. If $D=' 0$ ', it is a virtual point; if $D=$ ' 1 ', it is a normal old point. Unlike normal points that are automatically cancelled when they meet the ending endpoints, virtual points expire according to their $X$-extension bits. When the virtual point is first generated, the $X$-extension bits record the value of $d$. Assume the scanline moves to the right by $l$ after the current scanline has been processed, then the $X$-extension bits of all the virtual edges are updated by subtracting $l$ from themselves. If the value of the $X$ extension bits is less than or equal to zero, the corresponding virtual edge is cancelled. When multiple layers exist in the system, we set $d$ to the value of the largest value of minimum width/spacing requirement concerning this layer.

In order to minimize the number of virtual edges and to better accommodate virtual points in our method, we only generate virtual edges when:

(1) The right side of a forward true ending endpoint is outside the region. This is used for space checking, or

(2) The right side of a backward true ending endpoint is inside the region. This is used for width checking.

Thus in Figure 6, only $B$ and $D$ are generated as horizontal virtual edges, and only $A$ and $C$ are generated as vertical virtual edges. (Rotate the picture by 90 degree counterclockwise.) $Q$ will not be generated. Furthermore, we stipulate that a virtual edge never updates the $Y$-coordinate register of its layer.

\subsubsection{Flagging Errors}

To avoid flagging the same error many times, an error between current point and a latest point should only be flagged in the following two cases:

(1) If the current point is a true starting endpoint, or

(2) If the previous point was a starting endpoint and the current point is either a true point or a virtual point.

THEOREM 1 The design rule checking system described above will find all the design rule errors between adjacent edges and will not generate repetitive errors.

Proof We only prove the case for width checking between two adjacent horizontal edges $A B$ and $C D$ (see Fig. 7). As mentioned before in this paper, other types of checking can be transformed to width checking and can be proved similarly. Without losing generality, we assume $Y_{A B}<Y_{C D}$, $A B$ is a forward edge and $C D$ is a backward edge and there is a width error between these two edges.

Case $1 X_{A} \leq X_{C} \leq X_{B}$. The error will be found on any scanline that crosses the overlap part of these two edges, but the error will only be flagged when point $C$ is processed.

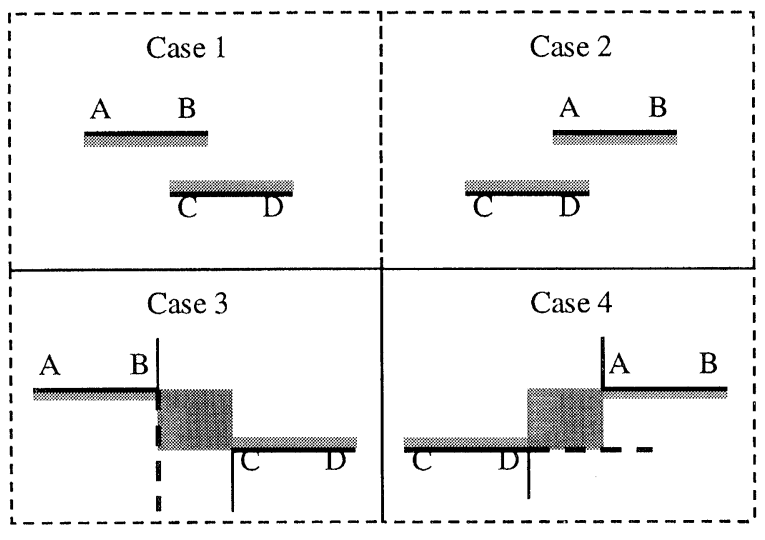

FIGURE 7 The four cases in Theorem 1. 
Case $2 X_{C} \leq X_{A} \leq X_{D}$. Similar to Case 1 .

Case $3 X_{A}<X_{B}<X_{C}<X_{D}$. Points $B$ and $C$ should be adjacent, otherwise these two edges are not adjacent. Consider the vertical edge $B$ is associated with. Since $B$ and $C$ are adjacent, $B$ can only be the ending endpoints of this vertical edge. For the same reason, $C$ can only be the starting endpoints of its vertical edge. Thus $B$ will generate a vertical virtual edge and an error will be produced by this virtual edge at $\left(X_{B}, Y_{C D}\right)$.

Case $4 X_{C}<X_{D}<X_{A}<X_{B}$. Points $A$ and $D$ should be adjacent, otherwise these two edges are not adjacent. In this case, the rectangle formed by $A$ and $D$ will be inside the region, thus $D$ will generate a horizontal virtual edge which will produce the error message at $\left(X_{A}, Y_{C D}\right)$.

Thus in all four possible cases, the design rule checking system gives all errors and no repetitive errors are given.

\subsubsection{Generating Intermediate Layers}

Sometimes intermediate layers are needed for further design rule checking. For example, a gate "layer" is usually involved in many design rules but is not explicitly a layer in the layout file. In such cases, special working layers are generated for checking several design rules. Such layers are often Boolean transformations on true design layers. Thus points can be selected using the techniques described in Section 3.3.1. Unlike traditional edge-based hardware systems, the intermediate layers generated here are in canonical order; thus it can be further processed while it is being generated.

\subsection{Exploiting Parallelism}

Each mask layer can be sliced in vertical directions when dealing with horizontal edge endpoints (Fig. 8 ) and in horizontal directions when dealing with vertical edge endpoints. Each slice can be indivi-
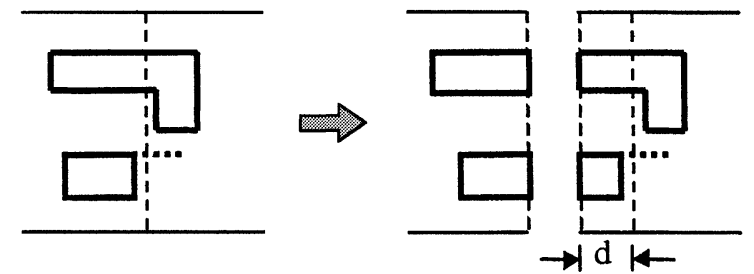

FIGURE 8 Mask slicing.

dually processed. Since all virtual edges crossing the cut disappear from the slice on the right, we need to extend each slice a length $d$ to ensure that these virtual edges are properly checked. Here $d$ is the maximum length of all the virtual edges, which is equivalent to the largest minimum width/spacing required in all the design rules. We thus expect a performance penalty of $d / s$, where $s$ is the width of each resulting slice.

Edge reconciliation does not impose a big problem for our system since errors are only checked along the scanline direction. No spurious error will be generated by our system. Repetitive errors will be generated in the overlapped region. They can be easily removed by "ORing" the errors collected from all slices.

\section{HARDWARE ARCHITECTURE}

Our hardware architecture is composed of one or more basic processing units (Fig. 3). Each basic processing unit (BPU) resembles the hardware architecture described in [1]. The Memory in Figure 3 gets the edge endpoints (i.e., new endpoints) from mask layers and the buffers store the "inherited" endpoints (i.e., old endpoints) from the previous scanline. Since the processing unit generates the old points for the next scanline while consuming the old points generated by the previous scanline, we keep two buffers. Buffer 0 is being read while buffer 1 is written and vice versa.

A Merge unit is used to maintain the scanline. Since both the data from the memory and from the buffer are in their canonical order, the merge unit does a simple two-way merge sort to generate the final data stream for processing. In addition, the 
merge sort unit also takes care of "retiring" old points and virtual points. As mentioned in Section 3.2 , old points are removed when they meet the ending endpoints. Each old point has the same representation as the corresponding ending endpoint except for the $P$ bit, which is the least significant bit in comparison; thus it will definitely meet the ending endpoint in the merge unit and it will be cancelled then. For virtual points, as mentioned in Section 3.3.3, the $X$-extension bits of the virtual points are updated in the merge unit, and virtual points are cancelled if $X$-extension bits are less than or equal to zero.

The checking unit is different for each BPU. Its basic structure is shown in Figure 5. For each layer, we need a set of counters and an additional subtractor. Thus the hardware cost grows roughly linearly with the number of layers we send to each checking unit. The only exception is the number of design rules involved. If all layers processed by a checking unit interact with each other, the number of design rules is roughly quadratic to the number of layers.

When an intermediate layer needs to be generated, the data from the checking unit will be fed into the memory of one or more subsequent BPUs. Temporary storage is required to store this intermediate layer. The merge unit of the subsequent processing units should be able to get the data from its own memory and from this temporary storage and does a preliminary merge sort to keep the new endpoints in canonical order.

\section{DESIGN RULES ANALYSIS}

Based on the hardware architecture described above, we have done a proof-of-concept implementation for the MOSIS submicron design rules [10]. In this section, we analyze the MOSIS submicron design rules and in the next section, we present our experiment results.

\subsection{Introduction to SCMOS Design Rules}

For this work, we focused on the MOSIS SCMOS_SUBM design rules, used for sub-micron fabrication. There are 27 SCMOS layers possible overall, but for a specific fabrication process, only a subset of the above layers are used. We concentrate here on the SCN4M_SUBM process for digital circuits. This process is a $0.4 \mu \mathrm{m} \mathrm{N}$-well process with 4 metal layers. Table I lists the related design rules for this process and the layers they involve. Each rule in Table I is broken down into several concrete design rule checks. For example, Rule 1 and Rule 2 are listed in detail in Table II. In all these rules, the largest minimum width/spacing in these rules is $18 \lambda$. This will impact the time that edges and points spend recirculating through the design rule checker. The values of minimum width/spacing specified in the rules are all multiples of $\lambda$ except Rule 5.2 and Rule 6.2 where $0.5 \lambda$ is used. This granularity impacts the total number of points that need to be processed for a given die size.

\subsection{Width Analysis}

We assume all the endpoints on the layout are on a $0.5 \lambda \times 0.5 \lambda$ grid. Since $\lambda=0.2 \mu \mathrm{m}$, the grid

TABLE I SCN4M related rules and layers

\begin{tabular}{|c|c|}
\hline Rule 1 - Well & $N$-well, $N$-high-voltage \\
\hline Rule 2 - Active & $\begin{array}{l}\text { Active, } N \text {-well, } N \text {-high-voltage, } \\
N \text {-plus-select, } P \text {-plus-select }\end{array}$ \\
\hline Rule 3 - Poly & Poly, active \\
\hline Rule 4 - Select & $\begin{array}{l}N \text {-plus-select, } P \text {-plus-select, gate, } \\
\text { contact, active }\end{array}$ \\
\hline Rule 5-Contact to poly & contact, poly, gate \\
\hline Rule 6 - Contact to active & contact, gate \\
\hline Rule 7 - Metal 1 & metal 1 , contact \\
\hline Rule 8 - Via 1 & via 1 , metal 1 , poly, active \\
\hline Rule 9 - Metal 2 & metal 2 , via 1 \\
\hline Rule 14 - Via 2 & via 2 , via 1 , metal 2 \\
\hline Rule 15 - Metal 3 & metal 3, via 2 \\
\hline Rule 21 - Via 3 & via 3 , metal 3 \\
\hline Rule 22 - Metal 4 & metal 4 , via 3 \\
\hline
\end{tabular}

TABLE II SCMOS_SUBM layout rules - well, active

\begin{tabular}{llc}
\hline Rule & \multicolumn{1}{c}{ Description } & $\lambda$ \\
\hline 1.1 & Minimum width of well & 12 \\
1.2 & Min spacing for wells of different potential & 18 \\
1.3 & Minimum spacing for wells of same potential & 0 or 6 \\
2.1 & Minimum width of active & 3 \\
2.2 & Minimum spacing between active & 3 \\
2.3 & Source/drain active to well edge & 6 \\
2.4 & Substrate/well contact active to well edge & 3 \\
2.5 & Min spacing between active of & 0 or 4 \\
& different implant & \\
\hline
\end{tabular}


spacing is $0.1 \mu \mathrm{m}$. If the maximum die width is $2 \mathrm{~cm}$, then we can have at most $2 \times 10^{5}$ points on a vertical line. Thus we use 18 bits to store the $X$ and $Y$-coordinates. For non-lambda design rules, the grid size required could be even smaller than $0.1 \mu \mathrm{m}$, resulting in a larger bit-width for $X$ and $Y$ coordinates.

Since there are 14 layers involved in the related design rules as shown in Table I, 4 bits are used to store layer information. Since the largest minimum width/spacing is $18 \lambda$ and the grid is $0.5 \lambda$, the largest value for $X$-extension bits would be 36 , so we use 6 bits to store it. From Figure 2, we need 31 bits for an edge endpoint. For simplicity, we use 32 bits for each endpoint and the one extra bit is given to the layer field since some intermediate layers might be generated on the fly.

\subsection{Layer Analysis}

Theoretically speaking, we could have sorted all the edge endpoints from all mask layers and sent them through a large checking unit to figure out all the design rule errors. However, as mentioned in Section 4, the hardware requirement grows more than linearly with the number of layers involved. As shown in Figure 5, each incoming endpoint's $Y$-coordinate is sent to a subtractor. If there are significantly more than 14 subtractors in our hardware, routing will be a problem since the $Y$ coordinate is 18 bits wide. Even if the routing were possible, we would expect a large delay. For this reason, our hardware design allows at most 5 layers to be checked by a basic checking unit.

We divide the layers into several groups and process them independently. From Table I, we derive the layer interaction graph shown in Figure 9. An edge is added if there is a design rule between these two layers. Our objective is to divide the graph into several cliques such that each clique has less than 5 layers and the duplicated layers are minimized.

We divide the groups as follows:

Group 1: Poly, Active, $N^{+}$-select, $P^{+}$-select. Intermediate layers $N$-active and $P$-active are generated.

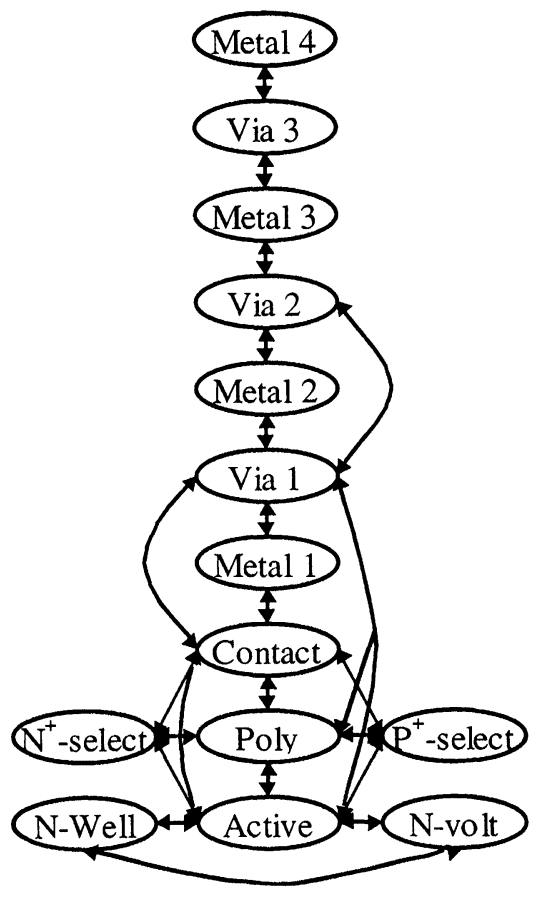

FIGURE 9 Layer interaction graph.

Group 2: $N$-active, $P$-active, $N$-well, $P$-well

Group 3: Poly, Active, Contact. Via 1

Group 4: Contact, Metal 1, Via 1, Metal 2, Via 2

Group 5: Via 2, Metal 3, Via 3, Metal 4

\section{EXPERIMENTAL RESULTS}

To test our ideas, we implemented a general merge unit as well as a check unit built specifically for Group 2 from the previous section. Since the merge unit is almost the same among different groups, we keep the merge unit and the checking unit on two separate FPGAs.

\subsection{Synthesis Results}

We used the Synopsys fpga_analyzer tool (1998.08) to generate .sxnf files from our VHDL input and we used Xilinx Foundation tools (V1.3) for the synthesis. We targeted our design at the speed of $33 \mathrm{MHz}$ for the merge and check units and specified tzhis information in the timing 
constraint file (.pcf file), where we listed this requirement for all the critical paths. After successful placement and routing, the synthesis results we get for the above design are listed in Table III. Most notably, our design fits in a Xilinx 4013 part and achieves the targeted clock rate for both units.

From the timing report of the synthesis result, we see that the bottleneck of the entire design is in the merge unit, where new endpoints and old points are compared. The check unit, on the contrary, can run much faster than the merge unit. Thus if a larger bit-width for $X$ and $Y$-coordinates is required, we expect that the clock rate will be slower because the comparison takes even longer time. However, since the number of logic levels in a comparator is $O\left(\log _{2} N\right)$, where $N$ is the bitwidth of a comparator, the delay increase on the critical path is marginal.

\subsection{Comparison Against Software}

To compare the hardware performance against software, we wrote a $\mathrm{C}$ program for our hardware implementation and tested it on several small CIF files (see Tab. IV). These CIF files were derived by synthesis and place-and-route of benchmark examples in BLIF format using the Octtools tool suite from UC Berkeley. We wrote another converter to convert the CIF files to our input format. Since the hardware we have implemented only performs design rule checking on $N$-well, $N$-highvoltage, $N$-active and $P$-active layers, we extracted from the CIF file the endpoints of these layers and sorted them in their canonical order. The resulting file is the input for both our software and hardware.

To optimize the software performance, we used an integer for each of the fields in the endpoint. We also tried to replace conditional jumps by Boolean operations wherever possible. We compiled our software on IRIX 6.3 IP32 system V with "ccO3" where global optimizations and procedure inlining are performed by the compiler. Then we used pixie to instrument the code based on basic blocks and prof-pixie-invocations to collect the data on total number of points being processed and software cycles and execution time in Table IV. We ran the software on an SGI O2 workstation with a single 4-way superscalar MIPS R10000 processor running at $150 \mathrm{MHz}$. Pixie uses the instruction scheduling model of this processor to obtain the data on software cycles and

TABLE III Synthesis results for merge and checking unit

\begin{tabular}{|c|c|c|c|c|c|}
\hline Unit & $\begin{array}{l}\text { Xilinx part } \\
\text { number }\end{array}$ & $\begin{array}{l}\text { CLB } \\
\text { used }\end{array}$ & $\begin{array}{l}\text { Flip- } \\
\text { flops }\end{array}$ & $\begin{array}{l}\text { Pipeline } \\
\text { stages }\end{array}$ & $\begin{array}{c}\text { Speed } \\
(\mathrm{MHz})\end{array}$ \\
\hline Merge & XC4013e-2 & 177 & 312 & 4 & 33 \\
\hline Check & $\mathrm{XC} 4013 \mathrm{e}-2$ & 262 & 419 & 4 & 33 \\
\hline
\end{tabular}

TABLE IV Experiment results (Benchmark Suit)

\begin{tabular}{|c|c|c|c|c|c|c|c|c|c|c|}
\hline $\begin{array}{l}\text { CIF } \\
\text { files }\end{array}$ & $\begin{array}{l}\text { \# of } \\
\text { scan } \\
\text { lines }\end{array}$ & $\begin{array}{l}\text { \# of } \\
\text { input } \\
\text { points }\end{array}$ & $\begin{array}{c}\text { \# of } \\
\text { total } \\
\text { points }\end{array}$ & $\begin{array}{l}\text { Ratio of } \\
\text { total pts/ } \\
\text { input pts }\end{array}$ & $\begin{array}{c}\text { \# of software } \\
\text { cycles } \\
\text { by pixie }\end{array}$ & $\begin{array}{l}\text { Cycle ratio } \\
\text { of software/ } \\
\text { hardware }\end{array}$ & $\begin{array}{l}\text { Software } \\
\text { runtime } \\
\text { by pixie }\end{array}$ & $\begin{array}{c}\text { Hardware } \\
\text { runtime }\end{array}$ & $\begin{array}{l}\text { Hardware } \\
\text { speedup } \\
\text { (150 MHz) }\end{array}$ & $\begin{array}{l}\text { Hardware } \\
\text { speedup } \\
(500 \mathrm{MHz})\end{array}$ \\
\hline $\mathrm{C} 17$ & 25 & 229 & 863 & $3.77: 1$ & 567327 & 657.39 & $2.71 \mathrm{~ms}$ & $0.0259 \mathrm{~ms}$ & 105 & 37.2 \\
\hline C432 & 52 & 1340 & 7859 & $5.86: 1$ & 4259747 & 542.02 & $22.12 \mathrm{~ms}$ & $0.2358 \mathrm{~ms}$ & 93.8 & 28.1 \\
\hline C499 & 37 & 1029 & 5506 & $5.35: 1$ & 3052718 & 554.43 & $15.53 \mathrm{~ms}$ & $0.1652 \mathrm{~ms}$ & 94.0 & 28.2 \\
\hline C880 & 44 & 1144 & 6482 & $5.67: 1$ & 3540159 & 546.15 & $18.24 \mathrm{~ms}$ & $0.1945 \mathrm{~ms}$ & 93.8 & 28.1 \\
\hline C1355 & 37 & 1029 & 5506 & $5.35: 1$ & 3052718 & 554.43 & $15.53 \mathrm{~ms}$ & $0.1652 \mathrm{~ms}$ & 94.0 & 28.2 \\
\hline C1908 & 40 & 1228 & 7017 & $5.79: 1$ & 3814853 & 543.66 & $19.68 \mathrm{~ms}$ & $0.2105 \mathrm{~ms}$ & 93.5 & 28.0 \\
\hline $\mathrm{C} 2670$ & 45 & 1517 & 8938 & $5.89: 1$ & 4806229 & 537.73 & $24.93 \mathrm{~ms}$ & $0.2681 \mathrm{~ms}$ & 93.0 & 27.9 \\
\hline C3540 & 40 & 1360 & 7829 & $5.76: 1$ & 4234389 & 540.86 & $21.85 \mathrm{~ms}$ & $0.2349 \mathrm{~ms}$ & 93.0 & 27.9 \\
\hline C5315 & 54 & 1518 & 8820 & $5.81: 1$ & 4775876 & 541.48 & $24.72 \mathrm{~ms}$ & $0.2646 \mathrm{~ms}$ & 93.4 & 28.0 \\
\hline C6288 & 48 & 1296 & 7311 & $5.64: 1$ & 3993868 & 546.28 & $20.55 \mathrm{~ms}$ & $0.2193 \mathrm{~ms}$ & 93.7 & 28.1 \\
\hline
\end{tabular}


execution time. The cycles per instruction (CPI) obtained by pixie is 0.868 , since pixie is unable to perform instruction scheduling between basic blocks, however, we expect true CPI to be slightly lower. The last column in the table shows the projected speedup over the software run on a MIPS R10000 processor, if it were clocked at $500 \mathrm{MHz}$ rather than $150 \mathrm{MHz}$.

From this table we can see, by using the hardware acceleration, we expect to achieve an average hardware speedup of 93.6X (excluding $\mathrm{C} 17$ since the number of endpoints in $\mathrm{C} 17$ is too small) over the software run on a $150 \mathrm{MHz}$ MIPS $\mathrm{R} 10000$ processor. We expect an average hardware speed up of 28.0 times (excluding $\mathrm{C} 17$ ) over the software run on a $500 \mathrm{MHz}$ MIPS R 10000 processor.

\subsection{I/O Requirement Analysis for Parallel Processing}

Using the parallelization method discussed in Section 3.4, we can connect multiple Basic Processing Units (Fig. 3) to the same I/O bus with each BPU configured to handle a different group of design rules. Assume a $66 \mathrm{MHz}$ 32-bit PCI interface for the $\mathrm{I} / \mathrm{O}$ bus, how many BPUs can we connect to this $\mathrm{I} / \mathrm{O}$ bus? This number is determined in part by how quickly each BPU is capable of processing new endpoints.

From Table IV we can see, in all but one benchmark $(\mathrm{C} 17)$, there is a more than 5 to 1 ratio between the total number of points each checking unit has to process compared to the input endpoints. Since the checking unit in each BPU processes one endpoint per clock cycle, the I/O requirement for each $\mathrm{BPU}$ is about $33 \mathrm{MHz} \times$ $32 \mathrm{bits} / 5=210 \mathrm{M}$ bps. Thus it would be possible for us to connect up to about 10 BPUs to this $210 \mathrm{M}$ bps I/O bus. This parallel limit is affected by the bit width of the endpoint and the clock rate of $\mathrm{I} / \mathrm{O}$ bus and BPU. If bit width increases or the clock rate of each BPU is improved, we will need a faster and wider $\mathrm{I} / \mathrm{O}$ bus to achieve the same parallelism.

\section{HARDWARE PROTOTYPING WITH PAMETTE}

The implementation and performance results presented thus far represent VHDL designs and simulations. We have also mapped our design to the PCI Pamette board [12] for hardware prototyping. The Pamette hardware architecture is somewhat different from our ideal hardware architecture described in Section 4. Figure 10 depicts how the design originally shown in Figure 3 is mapped to the four XC4010e FPGAs (i.e., LCA $0 \sim$ LCA3) of the Pamette. We put merge unit on LCA0 and check unit on LCA1. Instead of building two buffers for storing old points, we configure LCA2 to be a synchronous FIFO of 256 entries deep. As for LCA3, it is used to pass the old points generated from Check unit (LCA1) to the FIFO (LCA2).

We first configure the LCAs on the Pamette to download input endpoints into the SRAM0 on the board through PIF, the PCI interface. Then we reconfigure the board to our design to process these input points. The merge unit reads new endpoints from SRAM0 and old points from FIFO and outputs the current scanline point stream to check unit. The check unit performs rule check on this point stream, sends design rule errors to SRAM1 and writes the old points to the FIFO through LCA3. When the whole process is complete, the board is reconfigured again to read the errors from SRAM1 to the host machine.

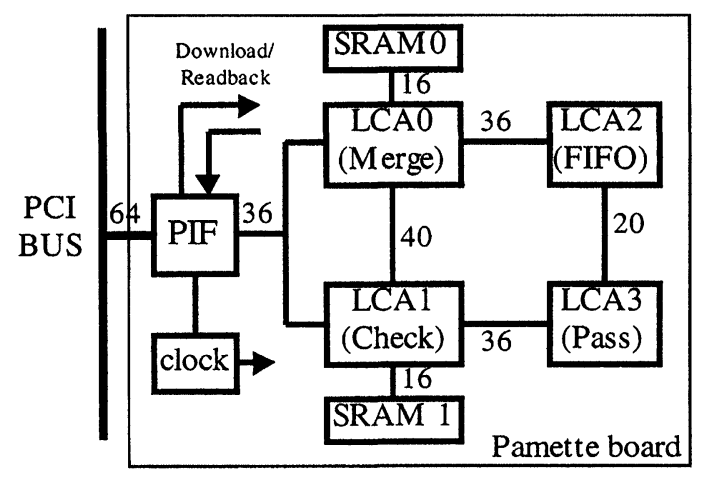

FIGURE 10 DRC block diagram on Pamette. 
From the Figure 10 we can see, the bus width between LCA3 and LCA2 is only 20 bits wide. Among these 20 bits, only 16 bits can be used freely. Since we need at least one bit for the "write_enable" signal on the FIFO, the representation for old points has to be less than or equal to 15 bits wide. Based on data width analysis of the benchmarks shown in Table IV, we tailored our original point representation (see Fig. 2) as follows. We use 7 bits for $Y$-coordinate, 2 bits for Layer, 1 bit each for Orientation and Direction and 4 bits for $X$-extensions. Since bit $P$ is always "1" for old points, we omit the bit $P$ in this representation.

The Pamette board has three modes [12]: static mode, transaction mode and miscellaneous mode. In our runtime implementation, we used static mode throughout the process and we used clkusr [13] to provide a $33 \mathrm{MHz}$ clock for the four LCAs. The correctness of the board has been verified by running through our entire suite of test examples.

\section{FUTURE WORK}

While the Pamette board is useful as a proof-ofconcept, we ultimately intend to build a specialized FPGA board for design rule checking. This board will have sufficient data buffering to handle large designs, and can also include several FPGAs in order to check a number of design rules in parallel.

This paper has focused on Manhattan structures in which all lines are either horizontal or vertical, and proposes that non-Manhattan structures be handled via software DRC. We are currently considering the hardware costs of handling special cases of non-Manhattan structures in hardware. For example, edges intersecting at 45-degree angles are the most commonly allowed nonManhattan structure. The constrained geometry of these 45-degree angles greatly eases their intersection calculations. In such a process, all the geometric structures can be comprised of either quadrilaterals or isosceles triangles, and the spacing computations require no floating point calculations; spacings can be inferred from manipulations of known wire lengths.

\section{CONCLUSIONS}

Because of the intrinsic similarity between different design rules [9], we have showed in this paper that hardware checking system for different design rules can be accommodated in a general scalable architecture. The variation between design rules of different fabrication processes makes configurable hardware an ideal candidate for the rulechecking unit in our general architecture. This paper describes and validates an edge-endpointbased geometry checking method for Manhattan structure, that is amenable to configurable hardware implementation. In comparison with previous edge-based methods, our technique uses a smaller datapath width, simplifies data flow control and easily handles the edge reconciliation problem.

As a verification of this general architecture, we analyzed the design rules of SCN4M_SUBM process from MOSIS and implemented the essential hardware for checking a subset of the design rules. The final hardware system runs at $33 \mathrm{MHz}$ and we expect to see a speedup of more than $28 \mathrm{X}$ over the conventional software run on the state-ofthe-art microprocessors.

\section{References}

[1] Eric C. Carlson and Rob A. Rutenbar, "A Scanline Data Structure Processor for VLSI Geometry Checking", IEEE Trans. Computer-Aided Design, CAD-6(5), September, 1987.

[2] Baker, C. M. (1980). "Artwork Analysis Tools for Integrated Circuits", MIT/LCS/TR-239, Master's Thesis, MIT.

[3] Seiler, L., "A Hardware Assisted Design Rule Check Architecture", Proc. 19th Design Automation Conf., June, 1982, pp. 235-238.

[4] Steven M. Rubin (1994). "Computer Aids for VLSI Design", 2nd edn., Addison-Wesley Publishing Company.

[5] Kane, R. and Sahni, S., "A Systolic Design Rule Checker", Proc. 21st Design Automation Conf., June, 1983, pp. $243-250$.

[6] Szymanski, T. G. and Van Wyk, C. J., "Space Efficient Algorithms for VLSI Artwork Analysis", Proc. 20th Design Automation Conf., June, 1983, pp. 734-739. 
[7] George E. Bier and Andrew R. Pleszkun, "An Algorithm for Design Rule Checking on a Multiprocessor", Proc. 22nd Design Automation Conf., June, 1985, pp. 299-304.

[8] Lauther, U., "An $O(N \log N)$ algorithm for Boolean mask operations", Proc. 18th Design Automation Conf., July, 1981 , pp. $555-562$.

[9] Lindsay, B. W. and Preas, B. T., "Design Rule Checking and Analysis of IC Mask Designs", Proc. 13th Design Automation Conf., June, 1976, pp. 301-308.

[10] MOSIS service, "MOSIS Scalable CMOS (SCMOS) Design Rules", Revision 7.2, http://www.mosis.org/New/ Technical/Designrules/dr-scmos $72 . \mathrm{html}$

[11] Mark Shand, "PCI Pamette: Generic PCI Board", http:// www.research.digital.com/SRC/pamette/

[12] Mark Shand, "PCI Pamette user-area interface for firmware V1.9", http://www.research.digital.com/SRC/ pamette/

\section{Authors' Biographies}

Zhen Luo got his B.S. degree from Computer Science and Technology Department of Peking University in 1996. He has been a Ph.D. student of Electrical Engineering Department of Princeton University ever since. His research interest include configurable computing, computer arithmetic, CAD for VLSI.
Margaret Martonosi has been an Assistant Professor of Electrical Engineering at Princeton University since 1994. Prior to that, Martonosi received M.S. and Ph.D. degrees from Stanford University and a B.S. degree with distinction from Cornell University. Her research interests lie at the hardware-software interface and most recently have focused on performance analysis techniques and their application to adaptive execution. She was a 1995 recipient of the NSF Career Award and a 1998 recipient of Princeton's Howard B. Wentz Award. She is a member of ACM and a senior member of IEEE.

Pranav Ashar received his M.S. and Ph.D. degrees in EECS from the University of California, Berkeley in 1990 and 91, respectively. He is currently the Department Head of the VLSI CAD Department at C\&C Research Labs, NEC in Princeton, NJ. His research interests are in the general area of VLSI CAD. He has a number of publications and patents to his credit in the areas of synthesis, verification and test. 

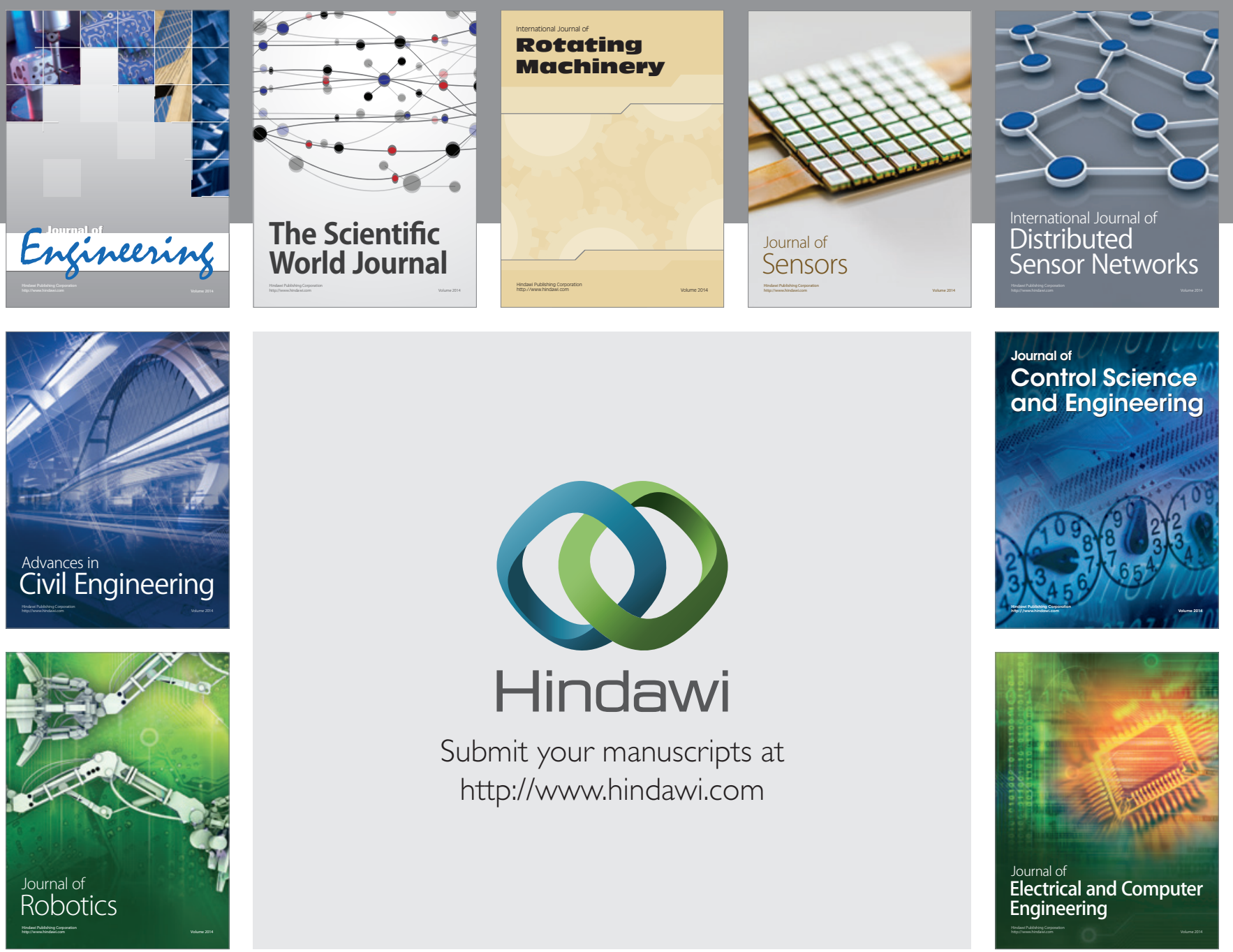

Submit your manuscripts at

http://www.hindawi.com
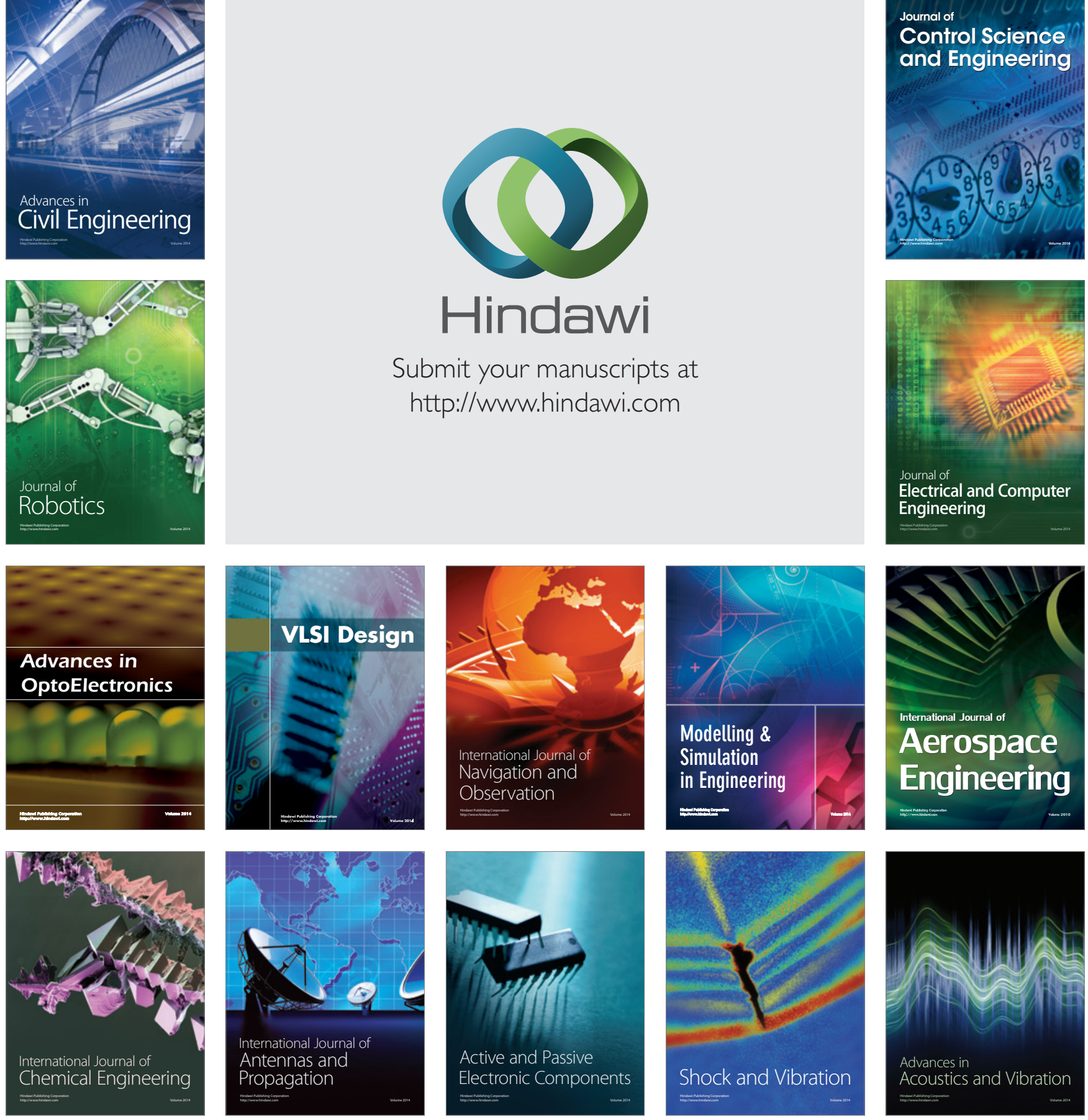